\title{
Mitochondrial health - essential information for nurses
}

\author{
Qiuhua Shen ${ }^{1}$, Elaina Knowles ${ }^{1}$, J John B Hiebert ${ }^{2}$, J anet D Pierce ${ }^{1}$ \\ 1. University of Kansas, School of Nursing, United States. 2. Lawrence Memorial Hospital, United States.
}

Correspondence: Qiuhua Shen. Address: 3901 Rainbow Blvd, Kansas City, KS 66160. Telephone: 913-945-5046.

Email: qshen@kumc.edu

Received: October 31, 2011

DOI : 10.5430/jnep.v2n2p162

Accepted: December 13, 2011 Published: May 1, 2012

URL: http://dx.doi.org/10.5430/jnep.v2n2p162

\begin{abstract}
One of the most important organelles in the body is the mitochondria. It is crucial for generating energy and producing free radicals. Mitochondrial health is essential to the prevention and treatment of diseases. However, cellular or molecular mechanisms such as mitochondrial dysfunction are not adequately addressed in the current essentials of the American Association of Colleges of Nursing (AACN) for all nursing programs. Thus, mitochondrial function content should be incorporated into all nursing curricula that are practice-based. In this article, we will review the anatomy and physiology of the mitochondria (i.e., coenzyme Q10), and the need to include mitochondrial health as a concept in nursing. We will present various diseases/conditions that are affected by mitochondrial dysfunction such as coronary artery disease, diabetes, and aging. In the future, with the expanding advances in the biological sciences, nurses need to learn more about cellular function particularly the mitochondria. Consequently, there should be a new emphasis on mitochondrial health by nurses in education and practice.
\end{abstract}

\section{Key words}

Mitochondrial health, Coenzyme Q10, Adenosine triphosphate, Nursing education

\section{I ntroduction}

With the completion of the human genome project, a greater emphasis has been put on genetics in nursing education and practice ${ }^{[1,2]}$. However, cellular and molecular mechanisms of various diseases that are important in patient health care have not received adequate attention by nurses ${ }^{[3]}$. Although cellular/molecular content is covered in most nursing programs, advanced information concerning cellular/molecular topics such as mitochondrial health, proteomics ${ }^{[4]}$, metabolomics ${ }^{[5]}$, Nrf2 ${ }^{[6]}$, titin ${ }^{[7]}$ are needed for nurses to keep up with rapid advances in clinical practice. Understanding the underlying cellular or molecular mechanisms of diseases could assist nurses in understanding the treatments that they administer, as well as preventive interventions to promote patient health.

Mitochondrial dysfunction has been implicated in most human diseases. Thus, mitochondrial health is an extremely important clinical concept for nurses to understand in order to provide optimal patient care. A mitochondrion is an essential organelle within all cells. Its chief role is the production of adenosine triphosphate (ATP), the currency of energy within cells. Various processes can damage mitochondria, leading to mitochondrial dysfunction, and these changes may 
contribute to the pathogenesis of various diseases ${ }^{[8]}$. Mitochondrial health is a new concept for nurses, which we believe should be introduced and emphasized in nursing education and practice.

The purpose of this article is to review mitochondrial function as a part of physiology and pathophysiology content in nursing curriculum and practice. Specifically, we will review mitochondrial function, the role of mitochondria in various diseases, and the importance of incorporating mitochondrial function content in nursing education and how this could be applied in practice.

\section{Physiology and pathophysiology in nursing education and practice}

Physiology and pathophysiology courses are required in many nursing programs, including associate degree, baccalaureate, masters in advanced practice such as nurse practitioner, clinical nurse specialist, or nurse anesthetist, and doctor of nursing practice. The focus of physiology is to help students understand the mechanical, physical and biochemical functions of humans in normal health ${ }^{[9]}$. Pathophysiology is the study of alterations in normal mechanical, physical, and biochemical functions under diseased states or abnormal conditions ${ }^{[10]}$. The combination of these normal and abnormal physiologic states provides a basis for nurses to understand preventive measures and disease treatments. Although incorporated into curricula, many cellular and molecular aspects in physiology and pathophysiology are often overly simplified in current nursing education. Cellular and molecular physiology is not sufficiently emphasized in the 2008 essentials of baccalaureate education for professional nursing practice ${ }^{[11]}, 2011$ essentials of master's ${ }^{[12]}$ and 2006 essentials of doctoral education ${ }^{[13]}$ for advanced practice nursing in American Association of Colleges of Nursing (AACN). In keeping pace with the rapid changes in medical knowledge, nurses should be knowledgeable and conversant with both integrative and molecular physiology. Thus, advanced cellular/molecular content such as mitochondrial health should be included in physiology and pathophysiology courses in nursing education at all levels that are clinically oriented or in pre-requisites to admission to those programs.

Lack of education at cellular or molecular levels puts nurses at a disadvantaged position when working with physicians whose training includes more in-depth education in cellular or molecular content. Nurses with masters or doctoral degrees may have better understanding of diseases, but still may not be equipped with adequate knowledge about diseases at the cellular/molecular level or mechanisms involved in the pathogenesis of diseases. Although genetics is an important aspect in nursing education, it is only a small component of cellular function.

To provide comprehensive evidence based practice, nurses should have a more thorough understanding of pathways or mechanisms of various diseases. For example, mitochondria play an important role in various diseases. However, nurses or even advanced practice nurses are usually not familiar with its anatomy and physiology, and its involvement in the pathogenesis of various diseases. The following content will briefly discuss mitochondrial function and its role in various diseases, as an example of how cellular/molecular physiology and pathophysiology would help nurses better understand disease processes affected by mitochondrial dysfunction.

\section{Mitochondrial function}

Mitochondria are multifunctional organelles in cells, and play essential roles in both survival and apoptosis of cells. They are the primary producers of ATP, which provides the essential energy for vital organs such as heart, brain, kidneys, and skeletal muscles ${ }^{[14]}$. Any alterations in mitochondrial function would have significant impact on the physiologic function of these systems that relate to the pathogenesis of many diseases such as heart disease and diabetes ${ }^{[15,16]}$. Therefore, maintaining mitochondrial health is essentially important in preventing diseases. By understanding mitochondrial function 
and the importance of maintaining mitochondrial health, nurses would be more knowledgeable in understanding diseases from cellular/molecular levels.

\subsection{Anatomy}

A mitochondrion has unique structural characteristics, as demonstrated in Figure 1. A mitochondrion consists of two double phospholipid layers, which are embedded with proteins. One is the outer membrane; the other is the inner membrane. Compared to the outer membrane, the inner membrane has many folds, called cristae. The productivity of cellular respiration is significantly enhanced by these folds with increased available surface area. The intermembrane space and the mitochondrial matrix are two distinct compartments that are divided by the double membranes. The intermembrane space is the narrow part between the outer and inner membranes and the mitochondrial matrix is enclosed by the inner membrane. There are hundreds of enzymes, mitochondrial ribosomes, DNA, and granules within the matrix ${ }^{[8,14]}$.

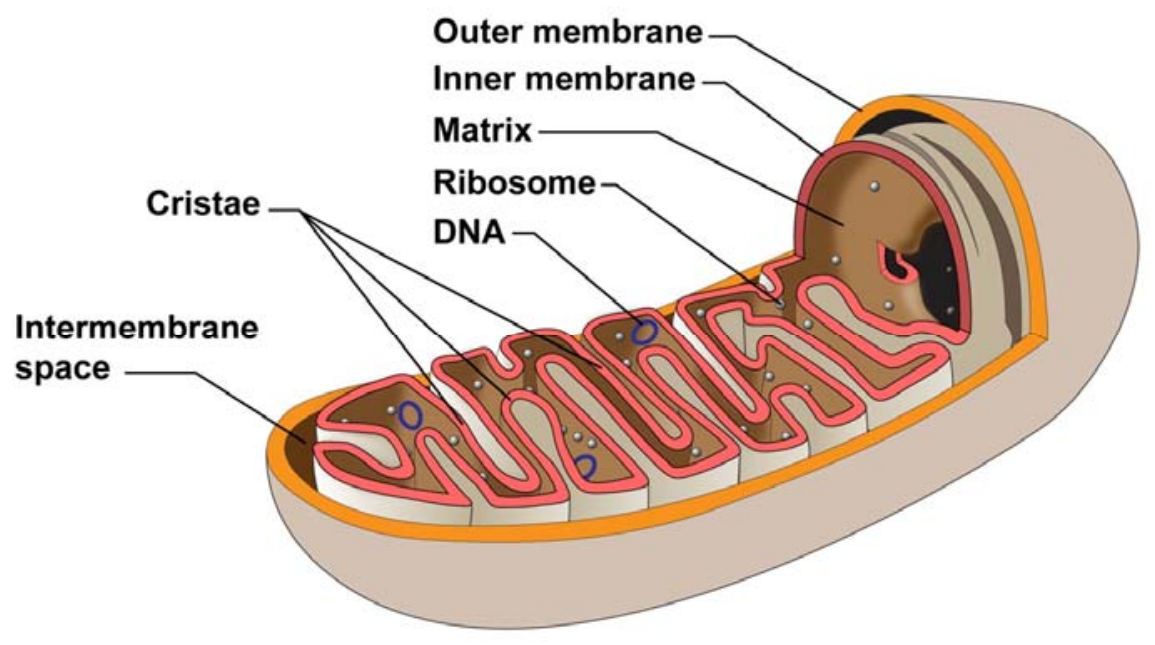

Figure 1. Anatomic Structure of a Mitochondrion

\subsection{Physiology}

Mitochondria are unique organelles, as they can produce their own DNA, RNA, and proteins. Mitochondria play a key role in energy metabolism that is essential for the survival and proliferation of eukaryotic cells. The primary job of mitochondria is to produce ATP, which converts caloric energy into molecular energy. In addition, mitochondria are also involved in electron transport and production of reactive oxygen species (ROS) ${ }^{[14]}$. The primary function of mitochondria varies by cell types. For example, the major function of mitochondria in an endothelial cell is to regulate the generation of oxidants for cell signaling. In contrast, the primary function of mitochondria in a cardiac myocyte is to generate ATP. Figure 2 demonstrates the five mitochondrial complexes in the electron transport chain.

Adenosine triphosphate (ATP) ATP is the molecular energy required for cells to function normally. Oxidative phosphorylation is the process that converts caloric energy into ATP. It begins when carbohydrates are metabolized into glucose and then into pyruvate in the cytoplasm via glycolysis. The pyruvate is transported into the mitochondrion and formed into acetyl CoA where it enters the citric acid cycle. This yields NADH and FADH2, which decrease equivalents utilized for electron transport. NADH is oxidized at complex I of the electron transport chain located within the mitochondrial inner membrane, while FADH2 is oxidized at complex II. Electrons are passed to coenzyme Q10 (CoQ10) 
from complex I and II. Reduced CoQ10 carries the electrons to complex III, which then passes the electrons to cytochrome c. In complex IV, electrons are removed from cytochrome c and transferred to oxygen, generating water. During electron transport, an electrochemical gradient is created by the protons being pumped across the inner membrane into the intermembrane space from the matrix. This energy is utilized to condense a molecule of inorganic phosphate with ADP at complex V, also known as ATP synthase, to yield ATP, which in turn is transported out of the matrix. Fats, on the other hand, undergo B-oxidation yielding acetyl CoA, which re-enters the citric acid cycle to generate substrates for electron transport $^{[14,17]}$.

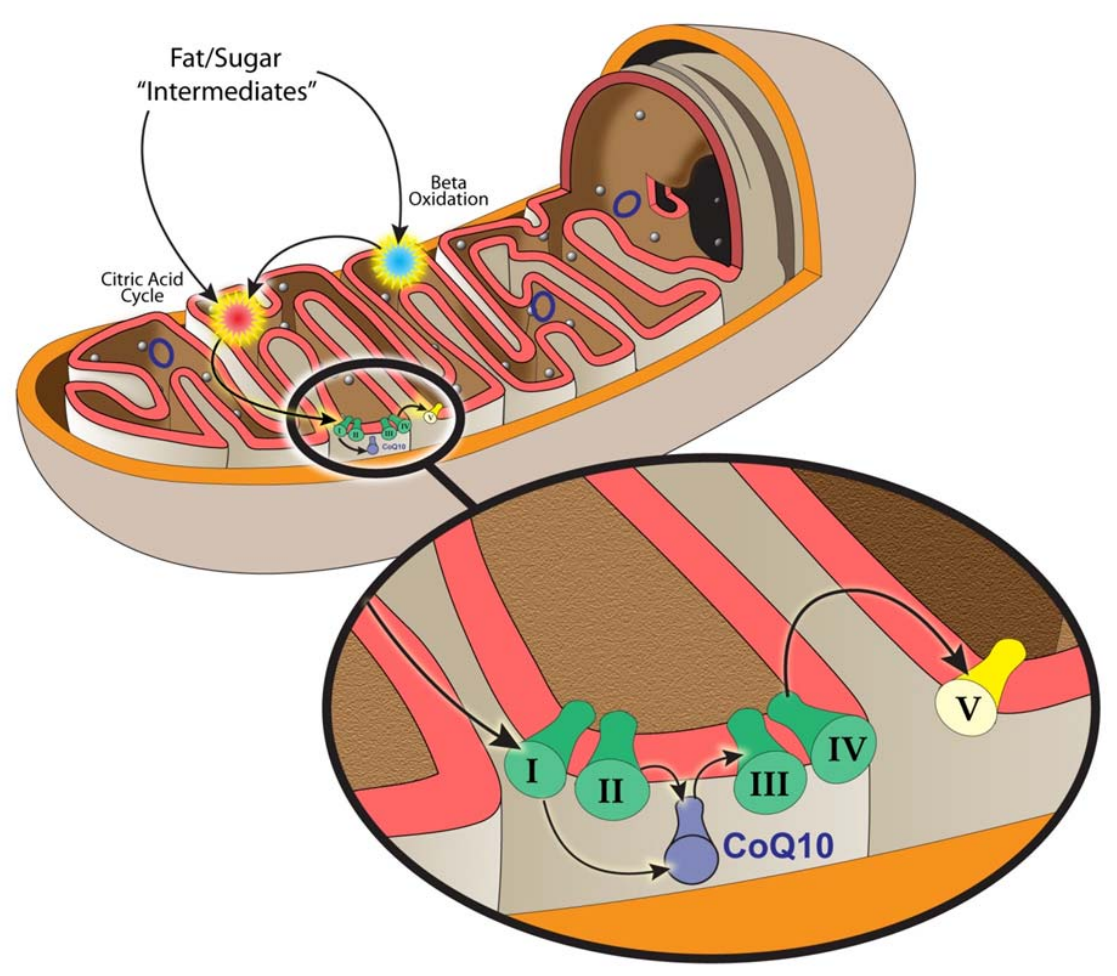

Figure 2. Five Mitochondrial Complexes in the Electron Transport Chain

Co-enzyme Q10 (CoQ10) CoQ10 is a lipid-soluble substance that is present mainly in the inner membrane of mitochondria. Ubiquinone is the fully oxidized state of CoQ10 and ubiquinol is the fully reduced form of CoQ10. Mitochondrial CoQ10 has two principal roles: 1) ATP generation, and 2) potent antioxidant. In its first role, CoQ10 works as an electron transporter from complexes I and II to complex III of the mitochondrial respiratory chain, which facilitates the formation of a proton gradient by pumping of $\mathrm{H}+$ across the membrane. ATP synthase uses the proton gradient to generate ATP. In its second role, CoQ10 is one of the most active scavengers for reactive oxygen species (ROS). The reduced form of CoQ10, ubiquinol, holds electrons loosely, and can easily give up one or two electrons to prevent DNA, lipids, and protein oxidation. Furthermore, CoQ10 can regenerate antioxidants, such as vitamin $\mathrm{E}^{[18]}$.

Reactive oxygen species are a group of highly reactive molecules that contain oxygen and unpaired electrons. They are a natural byproduct of the normal metabolism of oxygen that are generated in mitochondria during electron transport where electrons are added to oxygen $\left(\mathrm{O}_{2}\right)$, creating superoxide $\left(\mathrm{O}_{2}{ }^{-}\right)$. Superoxide is then converted by superoxide dismutase 2 (SOD2) to hydrogen peroxide $\left(\mathrm{H}_{2} \mathrm{O}_{2}\right)$, which can be further reduced to hydroxyl radicals $\left(\mathrm{OH}^{-}\right)^{[14]}$. Hydroxyl radicals can 
directly and indirectly damage DNA, lipids, and proteins. Excessive ROS can lead to apoptosis or programmed cell death when too much damage is caused to its mitochondria ${ }^{[19]}$.

\section{Mitochondrial role in various diseases}

Studies have shown that many diseases/conditions are affected by mitochondrial dysfunction ${ }^{[14,20-25]}$. When the mitochondrion does not function properly there are numerous mechanisms that are activated that can lead to cellular apoptosis. Most diseases and conditions are now associated with the cascades of programmed cell death. Thus, for this article we will discuss three conditions (heart disease, diabetes, and aging) and how these are influenced by mitochondrial health.

\subsection{Heart disease}

Alterations in mitochondrial functions may result in excessive oxidative stress, which is defined as the excessive production of reactive oxygen species, which outbalances the capability of antioxidants in the cells. Studies have shown that oxidative stress associated with cardiovascular disease (CVD) risk factors can lead to atherosclerotic lesions ${ }^{[26]}$, cardiometabolic disease, and hypertension ${ }^{[17]}$. Superoxide dismutase 2 (SOD2) is an enzyme in mitochondria that can transform toxic $\mathrm{O}_{2}{ }^{-}$into $\mathrm{H}_{2} \mathrm{O}_{2}$ and oxygen, and therefore protecting the cells. Decline in SOD2 activity can lead to an increase in cellular and mitochondrial oxidative stress, accompanying atherosclerotic lesion development under hypercholesterolemia. It was also found that exposure to CVD risk factors can decrease mitochondrial function and integrity, which enhances the impact of proatherogenic factors such as TNF-alpha, a proinflammatory cytokine on mitochondrial oxidative stress in the endothelium. This supports the hypothesis that decreased mitochondrial antioxidant levels can increase susceptibility to CVD development, thus showing the importance of the mitochondria in regulating its oxidant levels in order for proper cell function. Decreases in SOD2 levels are also found to inhibit vessel relaxation, contributing to hypertension, and act as a barrier against recovery from ischemic reperfusion ${ }^{[14,26,27]}$.

\subsection{Diabetes}

Mitochondrial dysfunction is closely associated with both obesity and the development of diabetes. Obesity is prevalent among prediabetes ${ }^{[28]}$. Excessive substrates are delivered to mitochondria due to increased fatty acid levels in prediabetes. This results in an increase in ROS production and intracellular fat accumulation, leading to mitochondrial dysfunction ${ }^{[29,30]}$. Mitochondria's ability to oxidize fat is compromised, causing interference with insulin signaling pathway and progressive worsening of insulin resistant state. This leads to the development of diabetes. Mitochondrial dysfunction also plays a role in diabetic complications. Diabetic retinopathy is one of the severe complications of diabetes, which occurs in almost $90 \%$ of patients suffering from diabetes within 20 years. Oxidative stress is one of the major factors contributing to diabetic retinopathy. This is evidenced by decreased SOD2 activities and increased $\mathrm{O}_{2}{ }^{-}$production in the retina and the capillary cells. There are significant alterations in mitochondria in these cells, including damaged mitochondrial DNA, decreased import of DNA repair enzymes, and abnormal expression of genes encoded by mitochondrial DNA. The number and mass of mitochondria are also reduced ${ }^{[1,32]}$.

\subsection{Aging}

Aging is a process of the accumulation of multidimensional changes in a person's life. From a biological perspective, several theories have been proposed to explain the mechanisms underlying age-related diseases and conditions. The free radical theory is one of the most popular theories, which proposes that ROS causes cumulative damage to biological macromolecules leading to irreversible cell damage and decline in functions. Under normal conditions, mitochondria are continuously undergoing dynamic changes in morphology by fusion and fission. These two processes are important mechanisms to maintain mitochondrial integrity, electrical and biochemical connectivity, modulating cellular redox status, turnover of mitochondria, and apoptosis. Interference to mitochondrial fusion and fission during aging could lead to abnormal mitochondrial morphology, with impaired integrity of mitochondrial DNA and biogenesis. Consequently, there 
is increased ROS production with aging. Mitochondrial DNA may be further compromised with the increased ROS generation, leading to a vicious cycle. Therefore, it is essential to maintain functional mitochondria to decrease age-related diseases, but there is an inevitable decline in mitochondria with aging ${ }^{[19,33]}$.

\section{I ncorporation of mitochondrial function content in nursing education and practice}

Considering the importance of mitochondrial health, we recommend the inclusion of information related to mitochondrial function and diseases in all levels of nursing education that are practice-focused. By emphasizing mitochondrial function in the nursing curriculum, undergraduate and graduate students will become more knowledgeable about the impact of mitochondrial health in the development of diseases. As presented above, some of the most prevalent diseases or pathophysiologic conditions have been found to have changes/alterations in mitochondrial function ${ }^{[14,20-25]}$. Examples of other common diseases/conditions related to mitochondria are presented in Table 1. The concept of mitochondrial health should be incorporated into the content of basic pathophysiology courses in nursing curriculum. Nurses should be conversant and familiar with the terminology and concepts related to mitochondrial health.

Table 1. Diseases/Conditions Associated with Mitochondrial Dysfunction ${ }^{[14]}$

\begin{tabular}{|r|r|}
\hline Cancer: & Breast \\
- & Prostate \\
• & Leukemia \\
Cardiovascular disease: \\
- & Hypertension \\
- & Coronary artery disease \\
- & Dystolic left ventricular dysfunction \\
- & Congestive heart failure \\
- & Pulmonary hypertension \\
- & Ischemial fibrillation \\
Diabetes & Mellitus \\
Neurologic Disorders: \\
- & Parkinson's disease \\
- & Alzheimer's disease \\
- & Cerebrovascular accidents (stroke) \\
- & Anoxite encephiles \\
Aging & \\
Obstructive sleep apnea \\
Diet \& Obesity \\
Exercise Limitation \\
\hline
\end{tabular}

\section{I mplication for nursing education, practice, and research}

Here are some suggested ways to include the topic of mitochondrial health into nursing programs. For a baccalaureate program, a prerequisite course such as molecular biology could be added before students can be admitted into the BSN program. The depth of curriculum content related to mitochondria should be advanced with the level of nursing education. 
For example, review of the anatomy and physiology of mitochondria should be sufficient for BSN programs. For an advanced practice degree such as a masters or doctoral program of nursing practice, more advanced information should be included. For example, for graduate students, curriculum content should include: mitochondrial complex 1-5 involving in the respiratory chain, the function of CoQ10 in generating ATP and reducing ROS, the differences between the oxidized (ubiquinone) and reduced form of CoQ10 (ubiquinol), and mitochondrial roles in both health maintenance as well as disease states.

By understanding the relationship of mitochondrial health to various diseases, nurses would better understand the underlying rationale of treatments to these diseases and be familiar with drugs that affect mitochondrial health. As an example, studies have shown that a deficiency in CoQ10 can exacerbate cardiometabolic, neurological, and other diseases including diabetes and cancer. However, supplementation of CoQ10 may alleviate myopathic symptoms. CoQ10 has been used as an adjunctive therapy for heart disease and for patients treated with statins ${ }^{[34]}$. By understanding role of CoQ10 in mitochondria, nurses would know that this is due to its antioxidant property in reducing endogenous oxidative stress and could better explain to patients who are taking CoQ10 supplements.

Mitochondrial health is one of many important research topics in the biomedical field. There are new therapeutic treatments being administered by nurses that specifically target mitochondrial function. For example, there are antioxidants (i.e. N-acetylcysteine) that are infused into patients to reduce free radical damage to the mitochondria ${ }^{[35]}$. Thus, if the nurse understands the mechanisms by which the mitochondria are maintained via antioxidants, they can explain to the patients and their families how the treatment works and the need to maintain mitochondrial health.

Nursing research is needed on how knowledge and understanding of mitochondrial function can guide nursing practice. There are nurse researchers currently studying the mechanisms that relate to the properties of mitochondria in order to optimize nursing interventions based on cellular function. These scientists are helping to pave the way for new interdisciplinary relationships with physicians, physiologists, molecular biologists, biostatisticians, and other healthcare professionals. Thus, future nursing research on mitochondrial health can also be collaborative and multidisciplinary efforts. Suggestions for clinical nursing practice and education studies include: 1) explore the effective ways to introduce mitochondrial health content into nursing curricula; 2) assess the impact of incorporation of mitochondrial health content into nursing education and practice on quality of patient care; and 3) find biomarkers to measure the effect of bedside nursing interventions on mitochondrial function. These few suggestions are only the beginning on how knowledge of mitochondrial health can improve nursing education and practice.

\section{Conclusion}

With the rapidly growing complexity of biosciences, nursing needs to increase knowledge concerning mitochondrial function and health. This begins with increasing content in nursing curriculum related to integrative and cellular physiology/pathophysiology. Many interventions, both pharmaceutical and non-pharmaceutical, currently impact the health of the mitochondria. We suggest that molecular biology be included in prerequisite courses for nursing undergraduate programs. In nursing curricula, cellular/molecular mechanisms such as mitochondrial health should be incorporated at all levels that are clinical in focus. Nurses in practice should have access to continuing education materials that focus on mitochondrial health. Thus, nurses would have improved cellular/molecular knowledge to provide optimal evidence based practice.

\section{Acknowledgement}

The Uniformed Services University of the Health Sciences (USU), 4301 Jones Bridge Rd., Bethesda, MD 20814-4799 is the awarding (award number: HU0001-11-1-TS09) and administering office. This research was sponsored by the TriService Nursing Research Program, Uniformed Services University of the Health Sciences; however, the information 
or content and conclusions do not necessarily represent the official position or policy of, nor should any official endorsement be inferred by, the TriService Nursing Research Program, Uniformed Services University of the Health Sciences, the Department of Defense, or the U.S. Government.

\section{Source(s) of support}

TriService Nursing Research Program, Uniformed Services University of the Health Sciences, the Department of Defense.

\section{Conflicting interest}

None

\section{References}

[1] Tonkin, E., Calzone, K., Jenkins, J., Lea, D., Prows, C., Genomic Education Resources for Nursing Faculty. J Nurs Scholarsh. 2011; 43: 330-340. PMid: 22034967 http://dx.doi.org/10.1111/j.1547-5069.2011.01415.x

[2] Calzone, K.A., Jerome-D'Emilia, B., Jenkins, J., Goldgar, C., Rackover, M., Jackson, J., et al., Establishment of the Genetic/Genomic Competency Center for Education. J Nurs Scholarsh. 2011; 43: 351-358. PMid:21981551 http://dx.doi.org/10.1111/j.1547-5069.2011.01412.x

[3] Davis, G.M., What is provided and what the registered nurse needs--bioscience learning through the pre-registration curriculum. Nurse Educ Today. 2010; 30: 707-712. PMid:20129721 http://dx.doi.org/10.1016/j.nedt.2010.01.008

[4] Ray, S., Reddy, P.J., Jain, R., Gollapalli, K., Moiyadi, A., Srivastava, S. Proteomic technologies for the identification of disease biomarkers in serum: advances and challenges ahead. Proteomics. 2011; 11: 2139-2161. PMid:21548090 http://dx.doi.org/10.1002/pmic.201000460

[5] Nagrath, D., Caneba, C., Karedath, T., Bellance, N. Metabolomics for mitochondrial and cancer studies. Biochim Biophys Acta. 2011; 1807: 650-663.

[6] Kang, K.W., Lee, S.J., Kim, S.G. Molecular mechanism of nrf2 activation by oxidative stress. Antioxid Redox Signal. 2005; 7: 1664-1673. http://dx.doi.org/10.1089/ars.2005.7.1664

[7] LeWinter, M.M., Wu, Y., Labeit, S., Granzier, H. Cardiac titin: structure, functions and role in disease. Clin Chim Acta. 2007; 375: 1-9. PMid:16904093 http://dx.doi.org/10.1016/j.cca.2006.06.035

[8] Cusimano, E.M., Knight, A.R., Slusser, J.G., Clancy, R.L., Pierce, J.D. Mitochondria: the hemi of the cell. Adv Emerg Nurs J. 2009; 31: 54-62. PMid:20118854

[9] Hall, J.E. Guyton and Hall textbook of medical physiology. (12th ed). Saunders. 2011.

[10] Kuma, V., Abbas, A.K., Fausto, N., Mitchell, R. Robbins basic pathology. (8th ed): Saunders. 2007.

[11] American Association of Colleges of Nursing. The essentials of baccalaureate education of professional nursing practice [Internet]. 2008. Available from: http://www.aacn.nche.edu/ccne-accreditation/standards-procedures-resources/baccalaureate-graduate/standards.

[12] American Association of Colleges of Nursing. The essentials of master's education in nursing [Internet]. 2011. Available from: http://www.aacn.nche.edu/ccne-accreditation/standards-procedures-resources/baccalaureate-graduate/standards.

[13] American Association of Colleges of Nursing. The essentials of doctoral education for advanced nursing practice [Internet]. 2006. Available from: http://www.aacn.nche.edu/ccne-accreditation/standards-procedures-resources/baccalaureate-graduate/standards.

[14] Svensson, O.L. Mitochondria: structure, functions and dysfunctions. (1st ed). Hauppauge, NY: Nova Science Pub Inc. 2011.

[15] Duncan, J.G. Mitochondrial dysfunction in diabetic cardiomyopathy. Biochim Biophys Acta. 2011; 1813: $1351-1359$.

[16] Bugger, H., Abel, E.D. Mitochondria in the diabetic heart. Cardiovasc Res 2010; 88: 229-240. PMid:20639213 http://dx.doi.org/10.1093/cvr/cvq239

[17] Krzywanski, D.M., Moellering, D.R., Fetterman, J.L., Dunham-Snary, K.J., Sammy, M.J., Ballinger, S.W. The mitochondrial paradigm for cardiovascular disease susceptibility and cellular function: a complementary concept to Mendelian genetics. Lab Invest. 2011; 91: 1122-1135. PMid:21647091 http://dx.doi.org/10.1038/labinvest.2011.95

[18] Genova, M.L. and Lenaz, G. New developments on the functions of coenzyme Q in mitochondria. Biofactors. 2011; 37: 330-354. PMid:21989973 http://dx.doi.org/10.1002/biof.168

[19] Cui, H., Kong, Y., Zhang, H. Oxidative stress, mitochondrial dysfunction, and aging. J Signal Transduct. 2012; $2012: 646354$.

[20] Kamp, D.W., Shacter, E., Weitzman, S.A. Chronic inflam mation and cancer: the role of the mitochondria. Oncology (Williston Park). 2011; 25: 400-413. PMid:21710835 
[21] Garcia-Roves, P.M. Mitochondrial pathophysiology and type 2 diabetes mellitus. Arch Physiol Biochem. 2011; 117: $177-187$. PMid:21671709 http://dx.doi.org/10.3109/13813455.2011.584538

[22] Schapira, A.H., Jenner, P. Etiology and pathogenesis of Parkinson's disease. Mov Disord. 2011; 26: 1049-1055. PMid:21626550 http://dx.doi.org/10.1002/mds.23732

[23] Galley, H.F. Oxidative stress and mitochondrial dysfunction in sepsis. Br J Anaesth. 2011; 107: 57-64. PMid:21596843 http://dx.doi.org/10.1093/bja/aer093

[24] Chen, C.M. Mitochondrial dysfunction, metabolic deficits, and increased oxidative stress in Huntington's disease. Chang Gung Med J. 2011; 34: 135-152. PMid:21539755

[25] Serviddio, G., Bellanti, F., Vendemiale, G., Altomare, E. Mitochondrial dysfunction in nonalcoholic steatohepatitis. Expert Rev Gastroenterol Hepatol. 2011; 5: 233-244. http://dx.doi.org/10.1586/egh.11.11

[26] Harrison, C.M., Pompilius, M., Pinkerton, K.E., Ballinger, S.W. Mitochondrial oxidative stress significantly influences atherogenic risk and cytokine-induced oxidant production. Environ Health Perspect. 2011; 119: 676-681. PMid:21169125 http://dx.doi.org/10.1289/ehp.1002857

[27] Lemieux, H., Semsroth, S., Antretter, H., Hofer, D., Gnaiger, E. Mitochondrial respiratory control and early defects of oxidative phosphorylation in the failing human heart. Int J Biochem Cell Biol. 2011.

[28] Garber, A.J. Obesity and type 2 diabetes: which patients are at risk? Diabetes Obes Metab. 2011.

[29] Brons, C., Jacobsen, S., Hiscock, N., White, A., Nilsson, E., Dunger, D., et al. Effects of High-Fat Overfeeding on Mitochondrial Function, Glucose and Fat Metabolism, and Adipokine Levels in Low Birth Weight Subjects. Am J Physiol Endocrinol Metab. 2011.

[30] Bournat, J.C., Brown, C.W. Mitochondrial dysfunction in obesity. Curr Opin Endocrinol Diabetes Obes. 2010 ; 17: 446-452. PMid:20585248 http://dx.doi.org/10.1097/MED.0b013e32833c3026

[31] Santos, J.M., Tewari, S., Goldberg, A.F., Kowluru, R.A. Mitochondrial biogenesis and the development of diabetic retinopathy. Free Radic Biol Med. 2011; 51: 1849-1860. PMid:21911054 http://dx.doi.org/10.1016/j.freeradbiomed.2011.08.017

[32] Szendroedi, J., Phielix, E., Roden, M., The role of mitochondria in insulin resistance and type 2 diabetes mellitus. Nat Rev Endocrinol. 2011.

[33] Seo, A.Y., Joseph, A.M., Dutta, D., Hwang, J.C., Aris, J.P., Leeuwenburgh, C. New insights into the role of mitochondria in aging: mitochondrial dynamics and more. J Cell Sci. 2010; 123: 2533-2542. PMid:20940129 http://dx.doi.org/10.1242/jcs.070490

[34] Won, R., Lee, K.H., Lee, B.H. Coenzyme Q10 protects neurons against neurotoxicity in hippocampal slice culture. Neuroreport. 2011; 22: 721-726. PMid:21849914 http://dx.doi.org/10.1097/WNR.0b013e32834acb8d

[35] Moradi, M., Mojtahedzadeh, M., Mandegari, A., Soltan-Sharifi, M.S., Najafi, A., Khajavi, M.R., et al. The role of glutathione-S-transferase polymorphisms on clinical outcome of ALI/ARDS patient treated with N-acetylcysteine. Respir Med. 2009; 103: 434-441. PMid:18993042 http://dx.doi.org/10.1016/j.rmed.2008.09.013 